\title{
So Many Nudges, So Little Time: Can Cost-effectiveness Tell Us When It Is Worthwhile to Try to Change Provider Behavior?
}

\author{
David Atkins, MD, MPH
}

Health Services Research and Development, Office of Research and Development, Department of Veterans Affairs, Washington, DC, USA.

J Gen Intern Med 34(6):783-4

DOI: $10.1007 / \mathrm{s} 11606-019-04871-5$

() Society of General Internal Medicine 2019

I nterest in behavioral economics has grown steadily within health care. Policy makers, payers, and providers now recognize that the decisions of patients and of their doctors frequently deviate from the strictly "rational" choices that classical economic theory would predict. For example, patients rarely adhere to the medication regimens or health behaviors that would optimize their health outcomes, and clinicians often make decisions that conflict with evidencebased recommendations or even the practices they profess to endorse. The groundbreaking work of psychologist Daniel Kahneman and his collaborator Amos Tversky raised attention to this field, which was accelerated by Kahneman's 2002 Nobel Prize in economics and his popular 2011 book "Thinking Fast and Slow" which reached a much broader audience. [1] Behavioral economics examines cognitive, psychological, and cultural factors that may influence how we make decisions, resulting in behavior that another Nobel laureate, economist Richard Thaler, has termed "predictably irrational." Principles from behavioral economics have been adopted to health care, including the role of heuristics (rules of thumb), the importance of framing, and the effects of specific cognitive biases (for example, overconfidence and status quo bias) [2]. These principles have been incorporated into interventions that seek to use these insights to change health-related behaviors - these include nudges, where systems are redesigned to make the preferred choice the default choice (for example, making generic versions the default in electronic prescribing); incentive programs that reward patients for taking their medications on schedule or getting preventive interventions like immunizations; and specific interventions aimed at how clinicians respond to information or make decisions.

The problem of antibiotic overprescribing would seem to be ripe for application of behavioral economics. Acute respiratory infections (ARIs) are one of the most frequent causes of medical visits. Since most ARIs are the result of viral infections, antibiotic prescriptions not only are ineffective but they

Published online March 15, 2019 also carry risks both to the individual in the form of antibiotic side effects, and to society by increasing antibiotic resistance. For over a decade, guidelines from CDC and professional societies have counseled caution in prescribing antibiotics in the outpatient setting, yet recent studies indicate overuse remains prevalent - in one recent study, $40 \%$ of patients presenting with ARI received antibiotics, and up to half had no clinical indication supporting antibiotic use [3]. Behavioral economics offers insights into why in practice many clinicians do not follow what they would otherwise endorse as good practice. Clinicians may overestimate their ability to diagnose serious infection, they may incorrectly assume patients will be unhappy if not given antibiotics or underestimate their adverse effects, and under time pressure they may default to reflex behaviors that favor prescribing. Individual habits or beliefs appear to play a strong role — a recent VA study documented a wide variation in clinician ARI practice, with individual antibiotic prescribing rates ranging from $40 \%$ to over $95 \%$ of patients presenting with ARI [4].

Despite the persistent problem of overprescribing, interventions can make a difference. A recent Cochrane Overview identified 8 systematic reviews and 44 RCTs identifying a variety of interventions that reduce prescribing without worsening patient satisfaction or symptom duration [5]. In this issue, Gong et al. [6] examine the costs and outcomes of different interventions in one of these trials, the Behavioral Economics to Improve Treatment of Acute Respiratory Infections (BEARI) [7]. BEARI was a cluster randomized trial comparing three approaches based on behavioral economic principles-(1) computerized decision support suggesting non-antibiotic alternatives; (2) accountable justification requiring clinicians to document reasons for prescribing antibiotics; and (3) peer comparisons, where periodic emails notify individual clinicians of how they compare to their peers in prescribing. Each of these interventions produced comparable reductions (16-18\%) in inappropriate prescribing over an 18month period. Gong used a Markov model to estimate the societal costs and outcomes compared to a baseline practice of provider education. Given comparable reductions in antibiotic use, it is not surprising that each intervention also improved health outcomes by similar amounts, primarily by reducing adverse effects of unnecessary antibiotics. When total societal costs are considered, savings from fewer 
prescriptions and emergency department (ED) visits for adverse reactions to antibiotics exceeded the costs of implementing the interventions. In cost-effectiveness parlance, this result-better health outcomes at lower costs - means any of the three strategies "dominates" the baseline state of traditional provider education.

One might be tempted to conclude that implementing these interventions is a win-win situation for all involved and that practices can choose any of the three alternatives as an improvement over current care. Unfortunately, there are many reasons that even a well-done analysis such as that of Gong et al. is not sufficient to change practice in healthcare. Often the problem is that the societal perspective recommended for most cost-effectiveness analyses aggregates all the costs and all the benefits of a policy regardless of who benefits or who pays. In reality, the parties who would have to implement the intervention (for example, a health care practice or health plan) are primarily interested in their own costs. Thus, an individual practice would bear the costs of implementing any of the interventions tested in BEARI but all the savings - from fewer antibiotics or ED visits - accrue to patients or their insurers. An interesting insight of Gong's analysis, however, is that the cost-savings did not depend on assumptions about reducing antibiotic resistance, an important but much more diffuse benefit to society. This raises the possibility that educational interventions aimed at patients could emphasize that avoiding unnecessary antibiotics can both keep them healthier and might save them money rather than simply appealing to a less tangible societal benefit. Alternatively, health plans or insurers who reap the savings might team with practices to help them implement these new measures.

One limitation of their analysis seems surprising, given the sensitivity of behavioral economics to non-economic factors that drive behavior. In calculating the costs of each intervention, they include the estimated time it might take in an office visit to implement the different interventions. Peer comparison, relying on intermittent emails, was the least burdensome while accountable justification was the most, taking twice as long as suggested alternatives. These differences, however, contribute little to the total costs of each strategy, which were driven more by costs of antibiotics and ED visits. In reality, clinicians' willingness to accept a new change in practice may be acutely sensitive to whether it adds 1 minute or 3 minutes to a common encounter. In an era where clinicians are feeling increasingly stressed for time, and ever more frustrated at their electronic health records and time spent documenting rather than caring for patients [8], we need to pay much more attention to how any new practice intervention affects patient flow and how it is received by clinicians. Deeper qualitative analysis can provide more complete insights on which of these interventions would be easiest to implement. If behavioral economics has taught us anything, it is that the experience of caring for patients - both its rewards and its frustrations - cannot be reduced to a matter of dollars and cents.

Corresponding Author: David Atkins, MD, MPH; Health Services Research and Development, Office of Research and Development, Department of Veterans Affairs, 810 Vermont Ave NW 10X, Washington, DC 20420, USA (e-mail: David.atkins@va.gov).

Publisher's Note: Springer Nature remains neutral with regard to jurisdictional claims in published maps and institutional affiliations.

\section{REFERENCES}

1. Kahneman D. Thinking Fast and Slow. New York: Farrah, Straus and Giroux, 2011.

2. Cho I and Bates D. Behavioral Economics Interventions in Clinical Decision Support Systems. Yearb Med Inform 2018; 27:114-21.

3. Havers FP, Hicks LA, Chung JR, Gaglani M, Murthy K, Zimmerman $\mathbf{R K}$, et al. Outpatient antibiotic prescribing for acute respiratory infections during influenza seasons. JAMA Netw Open 2018;1(2):e180243.

4. Jones BE, Sauer B, Jones MM, Campo J, Damal K, He T, et al. Variation in outpatient antibiotic prescribing for acute respiratory infections in the veteran population: a cross-sectional study. Ann Intern Med 2015;163:7380.

5. Tonkin-Crine SKG, Tan PS, van Hecke O, et al. Clinician-targeted interventions to influence antibiotic prescribing behavior for acute respiratory infections in primary care: an overview of systematic reviews. Cochrane Database of Systematic Reviews 2017, Issue 9. Art. No.: CD012252.

6. Gong CL, Zangwill KM, Hay JW, Meeker D, Doctor JN. Behavioral economics interventions to improve antibiotic prescribing for acute respiratory infections: a cost-effectiveness analysis. J Gen Intern Med. DOI: https://doi.org/10.1007/s11606-018-4467-x

7. Meeker D, Linder JA, Fox CR, Friedberg MW, Persell SD, Goldstein NJ, et al. Effect of Behavioral Interventions on Inappropriate Antibiotic Prescribing Among Primary Care Practices: A Randomized Clinical Trial. JAMA 2016: 315(6):562-70.

8. Gawande A. Why doctors hate their computers. New Yorker. https:// www.newyorker.com/magazine/2018/11/12/why-doctors-hate-theircomputers. Accessed 12 Nov 2018. 